\title{
ATRIBUTOS QUÍMICOS DO SOLO E CRESCIMENTO INICIAL DE PLANTAS DE MILHO EM LATOSSOLO ARENOSO COM ADIÇÃO DE CINZA DE CASCA DE ARROZ
}

\author{
Mateus Augusto Donegá ${ }^{1}$, Leandro Bochi da Silva Volk ${ }^{2}$, Antonio Nolla ${ }^{2}$, Thiago de Oliveira \\ Gaviolli $^{3}$
}

\footnotetext{
${ }^{1}$ Doutorando, Depto. de Produção Vegetal, ESALQ, USP, Av. Pádua Dias, 11, CEP:13418-060, Piracicaba-SP, Brasil. E-mail: donegamateus@gmail.com

${ }^{2}$ Professor Adjunto, Departamento de Ciências Agronômicas, Campus Regional de Umuarama, UEM, Estrada da Paca, s/n., CEP:87507-190, Umuarama-PR, Brasil.

${ }^{3}$ Engenheiro Agrônomo AGROCELLI, Avenida Independência, 1.629, CEP.: 85.950-000, Palotina - PR.
}

\section{RESUMO}

O objetivo deste trabalho foi verificar a influência da adição de cinza de casca de arroz (CCA) nas características químicas de um Latossolo Vermelho distrófico típico de textura arenosa e no desenvolvimento inicial de plantas de milho. O delineamento experimental utilizado foi o de blocos ao acaso com quatro repetições, sendo que cada parcela foi composta por um vaso com capacidade de $3 \mathrm{~L}$ de solo e com duas plantas de milho. Como tratamentos utilizou-se a adição de: $0,10,20,30,50$ e $100 \mathrm{Mg} \mathrm{ha}^{-1}$ de CCA. Após 45 dias da semeadura, as plantas foram coletadas para as avaliações biométricas (altura total, diâmetro do caule, massa fresca e seca da parte aérea e da raiz). Neste mesmo momento, o solo foi coletado para que fossem determinados os teores de $\mathrm{Ca}, \mathrm{Mg}, \mathrm{P}$ e $\mathrm{K}$, e os valores de $\mathrm{pH}$ em água e cloreto de cálcio. Observou-se que a aplicação de doses crescentes de CCA incrementou linearmente os valores de $\mathrm{pH}_{\mathrm{H} 2 \mathrm{O}}$ e $\mathrm{pH}_{\mathrm{CaCl} 2}$, e os teores de $\mathrm{K}, \mathrm{Ca}, \mathrm{Mg}$ e $\mathrm{P}$ no solo. A aplicação de doses crescentes de CCA propiciou, ainda, aumento na altura de plantas, diâmetro do caule e massa fresca e seca da parte aérea e do sistema radicular, sendo, o maior incremento observado entre as doses de 62 e $72 \mathrm{Mg} \mathrm{ha}^{-1}$.

Palavras-chave: Resíduo, Zea mays L., adubação alternativa

\section{SOIL CHEMICAL ATTRIBUTES AND INITIAL GROWTH OF CORN IN SANDY OXISOL WITH ADDITION OF RICE HUSK ASH}

\begin{abstract}
The aim of this study was to assess the influence of the addition of rice husk ash (RHA) on the chemical characteristics of a sandy Oxisol and initial development of corn plants. The experimental design was a randomized block design with four replications, andeach plot consisted of a $3 \mathrm{~L}$ capacity vase and two corn plants per vase. The treatments used were the addition of $0,10,20,30,50$ and $100 \mathrm{Mg} \mathrm{ha}^{-1}$ of RHA. After 45 days of sowing, plants were collected for the biometric evaluations (total height, stem diameter, fresh and dry weight of shoot and root). At this time, the soil was collected to determine the levels of $\mathrm{Ca}, \mathrm{Mg}, \mathrm{P}$ and $\mathrm{K}$, and $\mathrm{pH}$ values in water and calcium chloride. It was observed that the application of increasing doses of RHA linearly increased values and $\mathrm{pH}_{\mathrm{H}_{2} \mathrm{O}} \mathrm{pH}_{\mathrm{CaCl}}$, and the $\mathrm{K}, \mathrm{Ca}, \mathrm{Mg}$ and $\mathrm{P}$ in soil. The application of increasing doses of CCA has also provided an increase in plant height, stem
\end{abstract}


diameter and fresh and dry weight of shoot and root system. The highest increase observed between doses of 62 and $72 \mathrm{Mg} \mathrm{ha}^{-1}$.

Key words: Residue, Zea mays L., alternative fertilization

\section{INTRODUÇÃO}

Os solos oxídicos brasileiros, de modo geral, apresentam baixa fertilidade natural, $\mathrm{pH}$ ácido e alumínio trocável alto. Os solos da região noroeste do Estado do Paraná possuem como material de origem o arenito da formação Caiuá. Assim, são caracterizados por pertencerem às classes texturais areia e areia franca, apresentarem elevada acidez, bem como baixa capacidade de troca de cátions e teores de nutrientes (Sambatti et al., 2003).

Uma das alternativas para minimizar os custos de aplicação de insumos agrícolas nesses solos, principalmente em áreas de agricultura familiar, tem sido o emprego de resíduos agroindustriais e orgânicos, como condicionadores de solo e fonte de nutrientes. Resíduos alternativos viáveis são descritos na literatura especializada, como os da indústria sucroenergética (vinhaça, torta de filtro, bagaço), da indústria cítrica (polpa cítrica) e da indústria do aço (escória e pó de aciaria).

Presente diariamente na alimentação do brasileiro, o arroz tem produção estimada para a safra 2009/2010 de cerca de 12 milhões de toneladas (CONAB, 2010). Como consequência de seu beneficiamento, tem-se a produção elevada de casca de arroz (Della et al., 2001). Este subproduto pode ser utilizado para a geração de energia elétrica (Ueda et al., 2007), na construção civil, na fabricação de blocos e painéis (Santos, 1997), nas agroindústrias para geração de vapor e calor, nos processos de secagem e parboilização dos grãos de arroz (Della et al., 2001). Na maioria das vezes, as agroindústrias optam pelo uso da casca de arroz nos processos de geração de calor. O resíduo resultante desse processo é denominado de cinza de casca de arroz (CCA). O acúmulo e o destino inadequado desse resíduo trazem danos ao ambiente. Dessa forma, vêm sendo desenvolvidos trabalhos para a reutilização desse resíduo na indústria na fabricação de vidros, isolantes térmicos, tijolos prensados e na agricultura, como fonte de nutrientes e como corretivo de acidez do solo (Santos et al., 1995; Della et al., 2001).

As cinzas, de maneira geral, possuem teores variáveis de nutrientes (Mello, 1930). Possuem substâncias solúveis como os sulfatos, fosfatos e carbonatos de potássio e substâncias insolúveis como os carbonatos e fosfatos de cálcio e magnésio, além dos óxidos de ferro e manganês (D’utra, 1920).

A CCA tem potencial para ser utilizada na agricultura, em função das suas características físicas e químicas. Quanto às físicas, funciona como condicionador de solo e componente para a fabricação de substratos (Goepfert et al., 1980; Bellé \& Kampf, 1994; Minami, 1995; Nnabude \& Mbawu, 1999). Quanto as químicas, é recomendado como fonte de nutrientes para as plantas, pois contém elementos como fósforo, enxofre, potássio, magnésio, cálcio e silício (Kato \& Owa, 1997; Ueda, 2007). Na Tailândia, após a geração de energia, as indústrias fornecem aos produtores rurais esse resíduo para aplicação no solo (Ueda et al., 2007).

Assim, o objetivo deste trabalho foi verificar a influência da adição de cinza de casca de arroz nos valores de $\mathrm{pH}_{\mathrm{H}_{2} \mathrm{O}}$ e $\mathrm{pH}_{\mathrm{CaCl}}$, e nos teores de $\mathrm{K}, \mathrm{Ca}, \mathrm{Mg}$ e $\mathrm{P}$ no solo de um Latossolo Vermelho distrófico típico de textura arenosa, bem como no desenvolvimento inicial de plantas de milho. 


\section{MATERIAL E MÉTODOS}

O presente trabalho foi realizado no Laboratório de Solos do Campus Regional de Umuarama (CAU) da Universidade Estadual de Maringá (UEM), no ano de 2006.

O solo utilizado foi classificado como um Latossolo Vermelho distrófico típico (EMBRAPA, 2006) apresentando textura arenosa (Tabela 1). Este solo foi coletado na camada de 0 a $20 \mathrm{~cm}$ e após seco ao ar, foi homogeneizado e passado por uma peneira de malha de $2 \mathrm{~mm}$.

A CCA foi coletada junto a uma agroindústria local, após seu uso como fonte de energia em uma fornalha, e levada para laboratório para sua caracterização química, que encontra-se na Tabela 2.

$\mathrm{O}$ solo peneirado foi acondicionado em vasos com capacidade de três litros, utilizando-se os seguintes tratamentos, pela adição de: $0,10,20,30,50$ e $100 \mathrm{Mg} \mathrm{ha}^{-1}$ de CCA. Nesses vasos semeou-se milho de modo a serem obtidas duas plantas por vaso.

$\mathrm{O}$ delineamento experimental utilizado no experimento foi o de blocos ao acaso com quatro repetições, sendo que cada parcela foi composta por um vaso de $3 \mathrm{~L}$ contendo duas plantas.

Após 45 dias da semeadura, as plantas foram coletadas para as avaliações biométricas (altura total, diâmetro do caule, massa fresca e seca da parte aérea e da raiz). As amostras de solo de cada parcela, depois de coletadas, foram secas em estufa a $60^{\circ} \mathrm{C}$ e passadas em peneiras com malha de abertura de $2 \mathrm{~mm}$, para que posteriormente fossem determinados os teores de $\mathrm{Ca}, \mathrm{Mg}, \mathrm{P}$ e $\mathrm{K}$, e os valores de $\mathrm{pH}$ em água e cloreto de cálcio conforme metodologias descritas em EMBRAPA (1997).

Os dados foram submetidos à análise de variância. Para as relações entre os atributos químicos do solo e as doses de CCA foram realizadas análises de regressão linear, enquanto para as relações entre os atributos biométricos das plantas de milho e as doses de CCA foram realizadas análises de regressão polinomial.

\section{RESULTADOS E DISCUSSÃO}

Pelos resultados apresentados, verifica-se que a aplicação de cinza de casca de arroz alterou significativamente as características químicas (Tabela 3) e biométricas (Tabela 4) avaliadas.

Após 45 dias de aplicação de doses crescentes de CCA, houve incremento linear nos valores e $\mathrm{pH}$ em $\mathrm{H}_{2} \mathrm{O}$ e $\mathrm{CaCl}_{2}$ (Figuras $1 \mathrm{~A}$ e $1 \mathrm{~B}$ ) e nos teores de $\mathrm{K}, \mathrm{Ca}, \mathrm{Mg}$ e $\mathrm{P}$ (Figuras 1C, 1D, 1E e 1F), respectivamente.

Os valores de $\mathrm{pH}$ apresentaram correlação linear com as doses estudadas.

Tabela 1. Distribuição granulométrica do solo utilizado no estudo, na camada de 0 a $20 \mathrm{~cm}$.

\begin{tabular}{|c|c|c|c|}
\hline Areia & Silte & Argila & Classe textural \\
\hline & $\mathrm{g} \mathrm{kg}^{-1}$ & & \\
\hline 830 & 50 & 120 & Areia \\
\hline
\end{tabular}

Tabela 2. Caracterização química inicial do solo e da CCA utilizados no estudo.

\begin{tabular}{ccccccc}
\hline & \multicolumn{2}{c}{$\mathbf{p H}$} & $\mathbf{C a}$ & $\mathbf{M g}$ & $\mathbf{K}$ & $\mathbf{P}$ \\
\hline & $\mathbf{C a C l}_{\mathbf{2}}$ & $\mathbf{H}_{\mathbf{2}} \mathbf{O}$ & $---------\mathrm{cmol}_{\mathrm{c}} \mathrm{dm}^{-3}$ & ---------- & $\mathrm{mg} \mathrm{dm}^{-3}$ \\
Solo (0 a 20 cm) & 3,9 & 5,2 & 0,22 & 0,11 & 0,16 & 12 \\
$\mathbf{C C A}$ & 6,8 & 7,6 & 0,84 & 2,23 & 3,40 & 650 \\
\hline
\end{tabular}


Tabela 3. Valores de graus liberdade e quadrado médio do fator doses de CCA para as características químicas do solo $\mathrm{pH}_{\mathrm{H} 2 \mathrm{O}}, \mathrm{pH}_{\mathrm{CaCl} 2}, \mathrm{P}, \mathrm{K}, \mathrm{Ca}$ e $\mathrm{Mg}$.

\begin{tabular}{|c|c|c|c|c|c|c|c|}
\hline \multirow{2}{*}{$\begin{array}{l}\text { Causa de } \\
\text { variação }\end{array}$} & \multirow{2}{*}{$\begin{array}{c}\text { Graus } \\
\text { liberdade }\end{array}$} & \multicolumn{6}{|c|}{ Quadrado médio } \\
\hline & & $\mathrm{pH}_{\mathrm{H}_{2} \mathrm{O}}$ & $\mathrm{pH}_{\mathrm{CaCl} 2}$ & $\mathbf{P}$ & $\mathbf{K}$ & $\mathbf{C a}$ & $\mathrm{Mg}$ \\
\hline Doses de Cinza & 5 & $0,67 * *$ & $0,92 * *$ & $8656,35 * *$ & $85,86 * *$ & $19,12 * *$ & $546,92 * *$ \\
\hline Resíduo & 15 & & & & & & \\
\hline CV\% & & 1,13 & 1,19 & 21,22 & 11,57 & 13,75 & 6,05 \\
\hline
\end{tabular}

Tabela 4. Valores de graus liberdade e quadrado médio do fator doses de CCA para as características biométricas altura, diâmetro do caule (DIC), massa fresca da parte aérea (MFA), massa fresca de raiz (MFR), massa seca da parte aérea (MSA) e massa seca de raiz (MSR).

\begin{tabular}{|c|c|c|c|c|c|c|c|}
\hline \multirow{2}{*}{$\begin{array}{l}\text { Causa de } \\
\text { Variação }\end{array}$} & \multirow{2}{*}{$\begin{array}{c}\text { Graus } \\
\text { liberdade }\end{array}$} & \multicolumn{6}{|c|}{ Quadrado médio } \\
\hline & & Altura & DIC & MFA & MFR & MSA & MSR \\
\hline Doses de Cinza & 5 & $52,27 * *$ & $5,35 * *$ & $32,71 * *$ & $97,51 * *$ & $1,06 * *$ & $1,11 * *$ \\
\hline Resíduo & 15 & & & & & & \\
\hline CV\% & & 6,02 & 6,86 & 8,86 & 17,16 & 7,45 & 10,33 \\
\hline
\end{tabular}

Essa elevação de $\mathrm{pH}$ pode ser atribuída pela presença de carbonatos de potássio, cálcio e magnésio na CCA, que ao reagirem com a solução do solo liberam hidroxilas e neutralizam $\mathrm{Al}^{3^{+}}$e $\mathrm{H}^{0}$.

Outro fator que pode ter contribuído para a rápida elevação no $\mathrm{pH}$ foi que o solo utilizado no estudo apresentava textura arenosa e, assim, baixo poder de tamponamento de $\mathrm{pH}$, o que possibilitou que a partir da dose de $30 \mathrm{Mg} \mathrm{ha}^{-1}$, o pH adequado para o desenvolvimento das plantas já fosse alcançado (Rigau, 1960; Buedo, 1965; Malavolta, 1967). Evidenciase dessa forma, a possibilidade do uso da cinza como corretivo agrícola. Esse resultado corrobora com Santin \& Vahl (1985), Pauletto et al. (1990); Darolt et al. (1993) e Prado et al. (2002; 2003) que constataram efeito corretivo da acidez do solo com o uso de cinza vegetal.

Para o fósforo, o aumento na sua disponibilidade foi consideravelmente maior com a aplicação das doses de CCA usadas. Tal comportamento é salientado pelo coeficiente angular da equação de regressão para este elemento (Figura 1C), indicando que para cada unidade de CCA adicionada ao solo, o teor de fósforo aumentava na ordem de 1,27 vezes. Parte dessa elevação se deve ao elevado teor desse elemento na cinza (Tabela 2) e parte se deve a elevação do $\mathrm{pH}$ (Malavolta, 2006) também promovida pela adição de CCA que liberou parte do fósforo fixado nas argilas, contribuindo também para o aumento do teor de fósforo no solo. A aplicação da maior dose CCA (100 $\mathrm{Mg} \mathrm{ha}^{-1}$ ) foi a que proporcionou o maior incremento, chegando a elevar em cerca de 11 vezes os níveis desse nutriente no solo.

Para potássio, cálcio e magnésio, a dose de $100 \mathrm{Mg} \mathrm{ha}^{-1}$ de CCA, incrementou 
A

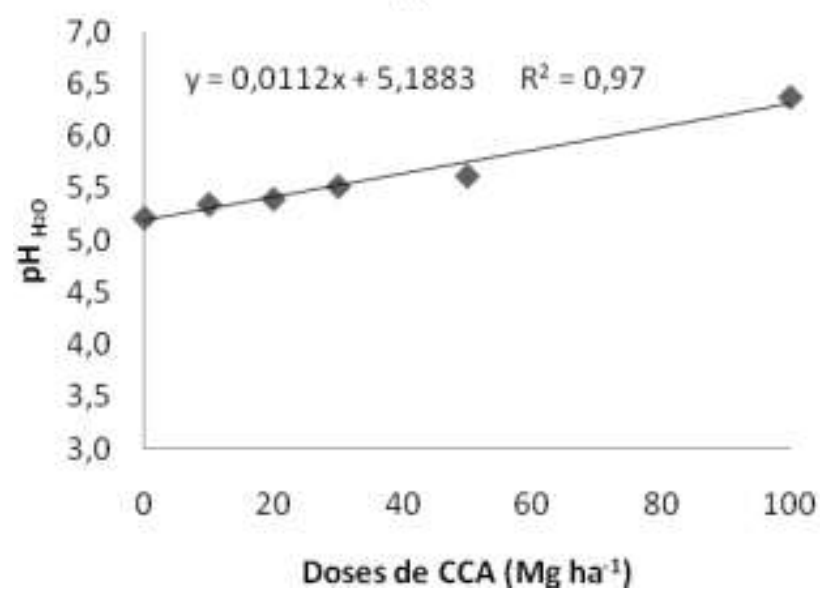

C

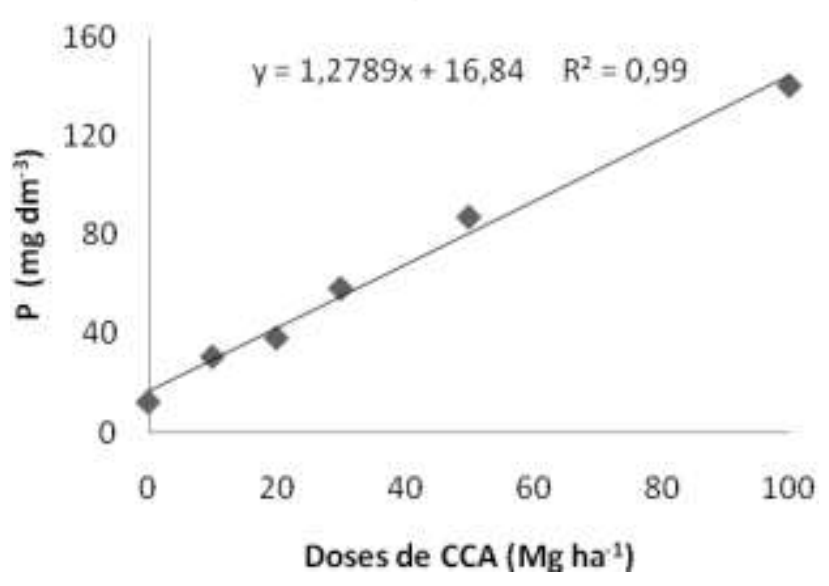

E

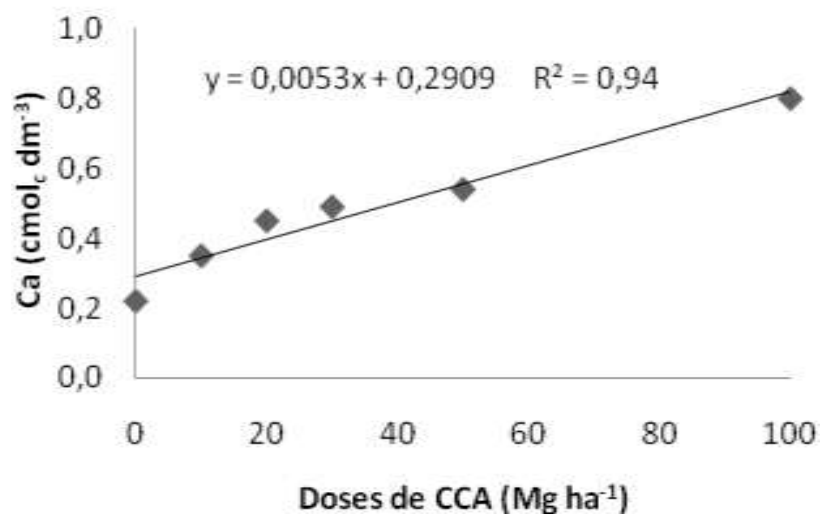

B

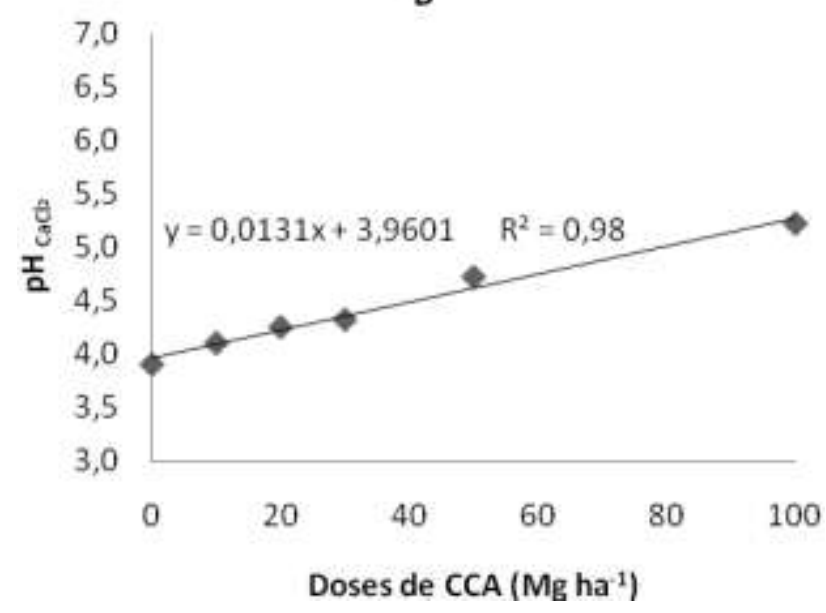

D

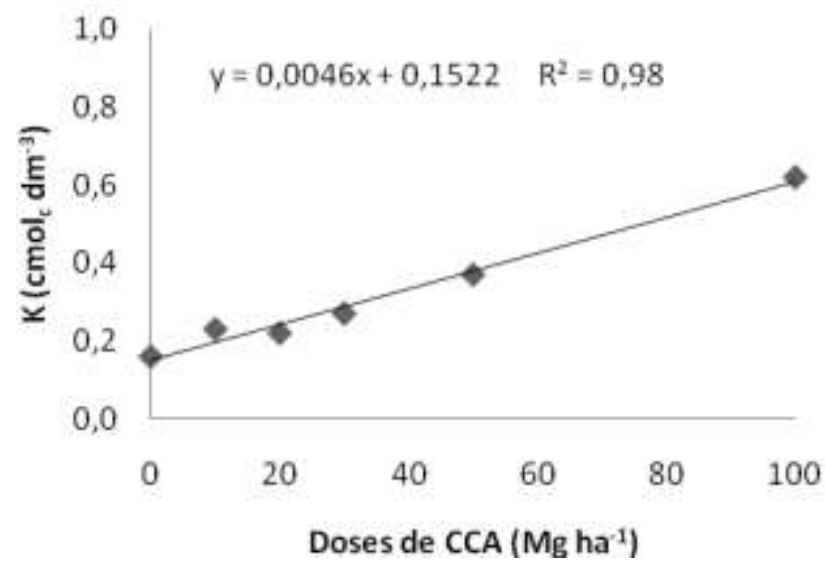

F

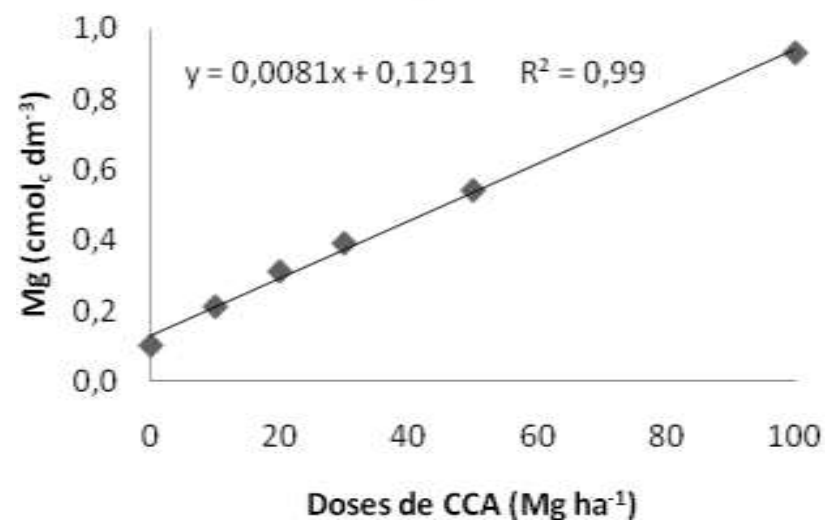

Figura 1. Valores de $\mathrm{pH}_{\mathrm{H} 2 \mathrm{O}}(\mathrm{A}), \mathrm{pH}_{\mathrm{CaCl}}(\mathrm{B})$, e teores de fósforo (C), potássio (D), cálcio (E) e magnésio (F) decorrentes da aplicação de doses de CCA em Latossolo Vermelho distrófico tipico. 

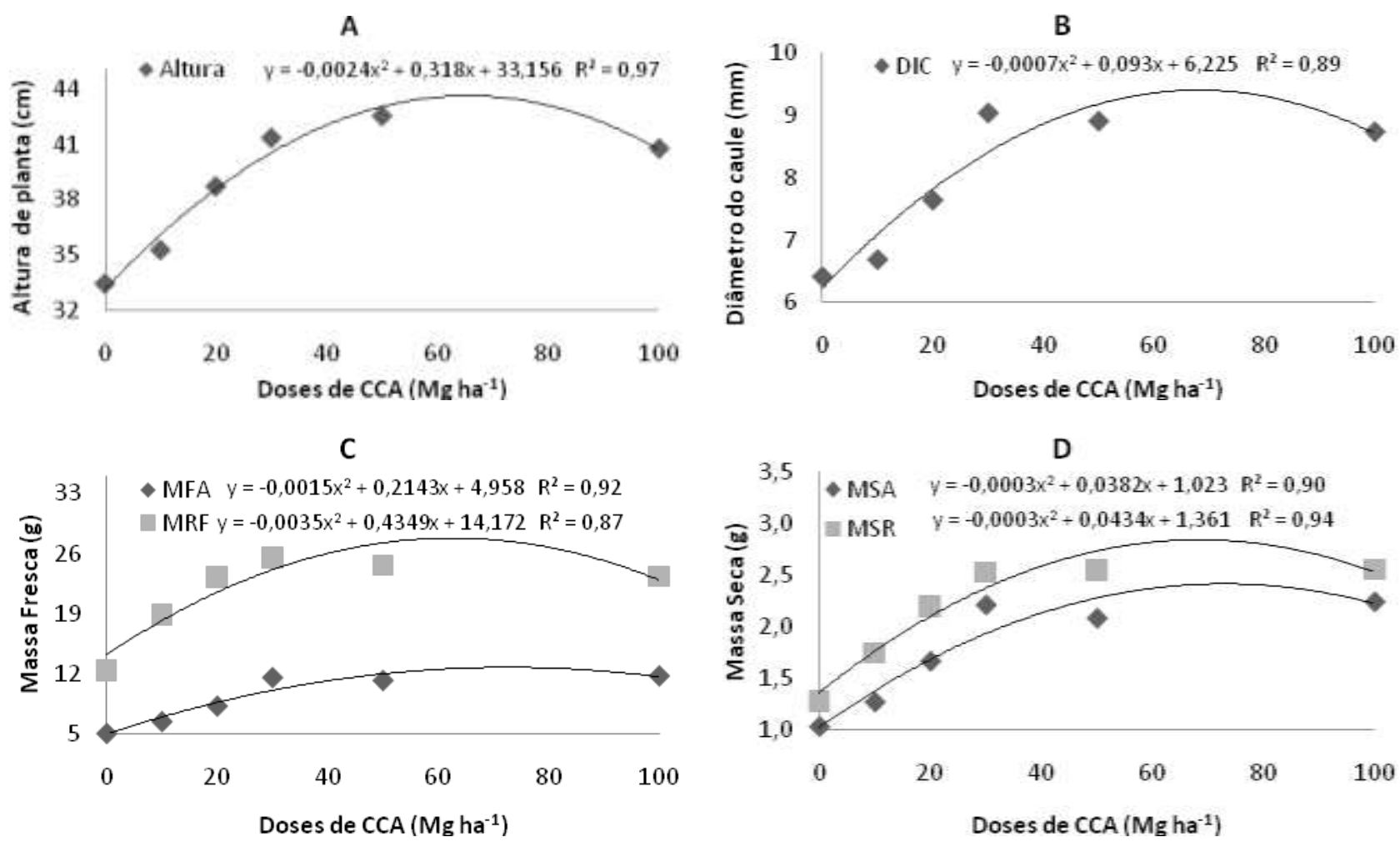

Figura 2. Altura (A), diâmetro do caule (DIC) (B), massa fresca da parte aérea (MFA), massa fresca de raiz (MFR), (C), massa seca da parte aérea (MSA), massa seca de raiz (MSR) (D) de plantas de milho em função das doses de cinza de casca de arroz.

os níveis desses elementos no solo em 3, 4 e 9 vezes, respectivamente, comparados com seus valores originais. $\mathrm{O}$ aumento na disponibilidade desses cátions está relacionado basicamente com a presença dos mesmos no material aplicado. Carbonatos de potássio, cálcio e magnésio são as principais fontes desses elementos nas cinzas. Assim como para o fósforo, a elevação nos valores de $\mathrm{pH}$ também pode ter contribuído para maior disponibilização desses nutrientes ao solo.

A utilização de cinza vegetal como fonte de nutrientes foi descrita por Goepfert et al.(1980); Santin \& Vahl (1985); Pauletto et al. (1990) e Prado et al. (2002; 2003).

Houve efeito positivo da aplicação de CCA para a altura, diâmetro do caule e massa fresca e seca da parte aérea e do sistema radicular das plantas de milho. Ocorreu incremento das características estudadas com a elevação das doses de CCA aplicadas, sendo que os maiores incrementos ocorreram entre as doses de 64 e $72 \mathrm{Mg} \mathrm{ha}^{-1}$. Havendo então a partir desses valores pequeno decréscimo nas médias das características biométricas.

A altura (Figura 2A) e diâmetro do caule (Figura 2B) apresentaram maiores médias na dosagem de $66 \mathrm{Mg} \mathrm{ha}^{-1}$ de CCA. Para massa fresca e massa seca da parte aérea (Figuras 2C e 2D), os maiores valores foram encontrados nos níveis de 72 e $64 \mathrm{Mg}$ $\mathrm{ha}^{-1}$ de CCA, respectivamente. Comportamento semelhante foi encontrado para a massa fresca e seca da raiz (Figuras $2 \mathrm{C}$ e 2D), pois, as médias foram máximas nas doses CCA de 62 e $72 \mathrm{Mg} \mathrm{ha}^{-1}$, respectivamente.Com base nesses resultados obtidos, pode-se inferir que, em média, a dose de $67 \mathrm{Mg} \mathrm{ha}^{-1}$, é a que pode proporcionar o maior incremento produtivo 
para plantas de milho em crescimento inicial. Esse incremento pode chegar a $47 \%$ quando se relaciona a dose média de $67 \mathrm{Mg}$ ha $^{-1}$ com a dose de $0 \mathrm{Mg} \mathrm{ha}^{-1}$. Almeida et al. (1988a; 1988b), trabalhando com cinza da casca de castanha do caju, nas culturas do sorgo e do milho, observaram incrementos na produção de grãos de 49 e $35 \%$ respectivamente, com relação ao controle.

Darolt et al. (1993) observaram o aumento significativo na massa e no diâmetro médio de cabeças de alface aplicando-se cinza vegetal.

\section{CONCLUSÕES}

As doses crescentes de cinza de casca de arroz adicionadas ao solo aumentaram linearmente o $\mathrm{pH}$ e os teores de $\mathrm{P}, \mathrm{K}, \mathrm{Ca}$ e $\mathrm{Mg}$. Dentre os atributos avaliados, o fósforo foi o que apresentou maior aumento do seu teor no solo com a aplicação da cinza de casca de arroz. A altura e o diâmetro do colmo das plantas de milho, bem como a massa fresca e seca da parte aérea e de raízes, apresentaram aumento com o uso de maiores doses de cinza de casca de arroz, com exceção da dose de $100 \mathrm{Mg} \mathrm{ha}^{-1}$. Doses entre 62 e $72 \mathrm{Mg} \mathrm{ha}^{-1}$ de cinza de casca de arroz em Latossolo arenoso foram as que promoveram os maiores valores para as atributos biométricos avaliados das plantas de milho.

\section{AGRADECIMENTOS}

À ZAELI LTDA. pela doação da cinza de casca de arroz.

\section{REFERÊNCIAS BIBLIOGRÁFICAS}

ALMEIDA, F.A.G.; FONTES, J.M.; ALMEIDA, F.C.G. 1988. Uso da cinza da Casca de Castanha de caju como fonte de fósforo para cultura do sorgo. Ciência Agronômica, 19: 67-72.
ALMEIDA， F.A.G.; FONTES， J.M.; ALMEIDA, F.C.G. 1988. Uso da cinza da Casca de Castanha de caju como fonte de fósforo para cultura do milho. Ciência Agronômica, 19: 73-78.

BELLÉ, S.; KAMPF, A.N. 1994. Utilização de casca de arroz carbonizada como condicionador hortícola para um solo orgânico.Pesquisa

Brasileira, 29: 265-271.

BUEDO, E.S. 1965. Fertilizantes Comerciales - Sus fuentes y uso . 1- Ed. $279 \mathrm{p}$

CONAB. 2010. Série histórica de produção. Disponível em http://www.conab.gov.br/conteudos.php $? \mathrm{a}=1252 \& \mathrm{t}=2$. Acessado em 30 de Julho de 2010.

D’UTRA, G.R.P. 1920. Adubos Chimicos. São Paulo. p. 74-76.

DAROLT, M.R. ; BLANCO NETO, V.A.; ZAMBON, F.R.A. 1993. Cinza Vegetal como fonte de nutriente e corretivo do solo na cultura da alface. Horticultura Brasileira, 11: 38-40.

DELLA, V.P.; KÜHN, I; HORTA, D. 2001. Caracterização de cinza de casca de arroz para o uso como matéria-prima na fabricação de refratário de sílica. Química Nova, 24: 778-782.

EMBRAPA 1997. EMPRESA BRASILEIRA DE PESQUISA AGROPECUÁRIA. Manual de métodos de análise de solo. 2. Ed. Rio de Janeiro, Ministério da Agricultura e do abastecimento. 212p.

EMBRAPA. 2006. EMPRESA BRASILEIRA DE PESQUISA AGROPECUÁRIA -. Centro Nacional de Pesquisa de Solos. Sistema brasileiro de classificação de solos. 2.ed. Rio de Janeiro. 306p.

GOEPFERT, C.F.; BENDJOYA, B.; POMPO, L.A. 1980. Efeito da cinza de carvão-de-pedra sobre o rendimento de bulbos de cebola (Allium cepa L.) e nas propriedades físicas e químicas do solo. 
Revista Brasileira de Ciência do Solo, 4: 96-99.

KATO, N.; OWA, N. 1997. Dissolution of slag fertilizers in a paddy soil and $\mathrm{Si}$ uptake by rice plant.Journal of Soil Science and Plant Nutrition, 43: 329341.

MALAVOLTA, E. 2006. Manual de nutrição mineral de plantas. São Paulo. Ceres. 638 p.

MALAVOLTA, E. 1967. Manual de química agrícola, adubos e adubações. 2Ed. São Paulo, Agronômica Ceres, 606p.

MELlO, P. C. 1930. Classificação e Análises de Adubos. Campinas. Instituto Agronômico, 25-27p.

MINAMI, K. 1995. Produção de mudas de alta qualidade em horticultura. São Paulo, $135 \mathrm{p}$.

NNABUDE,P.C.; MBAGWU, J.S.C. 1999. Soil water relations of a Nigerian typic haplustult amended with fresh and burnt rice-mill wastes. Soil \& Tillage Research, 50: 207-214.

PAULETTO, E. A.; NACHTIGALL, G. R.; GUADAGNIM, C. A. 1990. Adição de cinza de casca de arroz em dois solos do município de Pelotas-RS. Revista. Brasileira de. Ciência do. Solo, 14: 255-258.

PRADO, R.M.; CORREA, M.C. M.; PEREIRA, L.; CINTRA, A.C.O.; NATALE, W. 2003. Cinza da indústria de cerâmica na produção de mudas de goiabeira: Efeito no crescimento e na produção de matéria seca. Revista de Agricultura, 78: 25-35.

PRADO, R.M.; CORREA, M.C. M.; PEREIRA, L.; CINTRA, A.C.O.; NATALE, W. 2002. Efeito da cinza de indústria de cerâmica no solo e na nutrição de mudas de goiabeira. Acta Scientiarum (UEM), 24: 1493-1500.

RIGAU, A. 1960. Los Abonos - Su preparacion y emplego. 2 - ed. Barcelona, $80 \mathrm{p}$.

SAMBATTI, J.A.; SOUZA JÚNIOR, I.G.;
COSTA, A.C.S; TORMENA, C. 2003. Estimativa da acidez potencial pelo método do pH SMP em solos da formação Caiuá-Noroeste do Estado de Paraná. Revista Brasileira de Ciência do Solo, 27: 257-264.

SANTIN, M. J.; VAHL, L.C. 1985. Aproveitamento da cinza de casca de arroz como corretivo de acidez e da fertilidade do solo. Pelotas. In: Relatório CNPq.

SANTOS, J.A.G.; MOREAU, A.M.S.S.; REZENDE, J.O; COELHO, I.A.; SILVA, E.; CRUSOÉ,G. 1995. Avaliação do Potencial Corretivo da Cinza, Oriunda de Biomassa Vegetal, Comparada ao Calcário. In: CONGRESSO BRASILEIRO DE CIÊNCIA DO SOLO, 25. Resumos Expandidos, 2: 1148-1150.

SANTOS, S. 1997. Estudo da viabilidade de utilização de cinza de casca de arroz residual em argamassa e concretos. Dissertação (Mestrado em Engenharia) Universidade Federal de Santa Catarina, Florianópolis, 113p.

UEDA, T.; KUNIMITSU, Y.; SHINOGI, Y. 2007. Potencial conflicts for reuse of rice husk in Thailand. Paddy Water Environ, 5: 123-129. 